\title{
Uniqueness result for a fractional diffusion coefficient identification problem
}

Fadhel Jday ${ }^{1,2}$ and Ridha Mdimagh 1,3*

\section{"Correspondence:}

rmothman1@uj.edu.sa;

ridha.mdimagh@lamsin.rnu.tn; ridha.mdimagh@gmail.com

${ }^{1}$ Inverse Problems, LAMSIN, Ecole Nationale d'Ingénieurs de Tunis,

University of Tunis El Manar, Tunis Belvédère, Tunisia

${ }^{3}$ Mathematics Department, Faculty

of Science and Arts, Khulais,

University of Jeddah, Jeddah, Saudi

Arabia

Full list of author information is

available at the end of the article

\begin{abstract}
In this paper, we establish an identifiability result for the coefficient identification problem in a fractional diffusion equation in a bounded domain from the observation of the Cauchy data on particular subsets of the boundary.
\end{abstract}

MSC: 35R30; 35R11; 58J99

Keywords: Inverse problems; Fractional diffusion equation; Partial data; Uniqueness result

\section{Introduction}

Let $\Omega$ be a smooth bounded domain of $\mathbb{R}^{d}(d \geq 3)$ with boundary $\partial \Omega \in \mathcal{C}^{\infty}$, and let $T>0$ be a fixed real number. Our inverse problem consists in determining the potential $q:=q(x)$ via the fractional diffusion equation from given Cauchy data on particular subsets of the boundary. This problem is presented by the following equations:

$$
\left(P_{q, f}^{\alpha}\right) \quad \begin{cases}\partial_{t}^{\alpha} u-\Delta_{x} u+q(x) u=0 & \text { in } \Omega_{T}:=\Omega \times(0, T), \\ u(x, 0)=u_{0}(x) & \text { in } \Omega, \\ u=f & \text { on } \Sigma_{T}:=\partial \Omega \times(0, T),\end{cases}
$$

where $\partial_{t}^{\alpha} u$ represents the fractional Caputo time derivative of order $0<\alpha<1$ defined by equation (3.7). We assume that the coefficient $q \in L^{\infty}(\bar{\Omega}), f \in C^{1}\left([0 ; T] ; H^{\frac{3}{2}}(\partial \Omega)\right)$, and $u_{0} \in L^{2}(\Omega)$.

The classical diffusion-advection equation does not often describe the abnormal diffusion (e.g., for the problem of the soil scatter field data [1]) and that the fractional diffusion equation is used as a model equation for this problem and for many physical phenomena such as the diffusion of material in heterogeneous media, the diffusion of fluid flows in inhomogeneous anisotropic porous media, turbulent plasma, the diffusion of carriers in amorphous photoconductors, the diffusion in a turbulent medium flow, a percolation model in porous media, various biological phenomena, and finance problems (see [9]).

The inverse coefficients problems associated with the system $\left(P_{q, f}^{\alpha}\right)$ were investigated by many authors for the parabolic case $([8,12,13], \ldots)$ when $\alpha=1$ and for the hyperbolic case $([3-6,23], \ldots)$ when $\alpha=2$. Few works exist dealing with this type of problem in the fractional case $(0<\alpha<1)$. In [11] the authors, in the one-dimensional case, by Dirichlet boundary measurements determined the fractional order $\alpha$ and a time-independent

(c) The Author(s) 2019. This article is distributed under the terms of the Creative Commons Attribution 4.0 International License (http://creativecommons.org/licenses/by/4.0/), which permits unrestricted use, distribution, and reproduction in any medium, provided you give appropriate credit to the original author(s) and the source, provide a link to the Creative Commons license, and indicate if changes were made. 
coefficient. Using pointwise measurements of the solution over the entire time span, the authors in [16] identified the fractional order $\alpha$ for the case $d \geq 2$. Using a specifically designed Carleman estimate, the authors in $[10,25]$ derived a stability estimate of a zeroorder time-independent coefficient, with respect to partial internal observation of the solution in the particular case where $d=1$ and $\alpha=\frac{1}{2}$. In [14] the authors proved a unique determination of a time-dependent parameter appearing in the source term or in the zeroorder coefficient from pointwise measurements of the solution over the whole time interval. By performing a single measurement of the Cauchy data on the accessible boundary, the authors in [15] gave identifiability and local Lipschitz stability results to solve the inverse problem of identifying fractional sources. The authors in [19] proved a uniqueness result for the time-independent coefficients using the Dirichlet-to-Neumann operator obtained by probing the system with inhomogeneous Dirichlet boundary conditions of the form $\lambda(t) g(x)$, where $\lambda$ was a fixed real-analytic positive function of the time variable. In [17] the authors proved the uniqueness in the inverse problem of determining the smooth manifold (up to an isometry) and various time-independent smooth coefficients appearing in their equation from measurements of the solution on a subset of the boundary at fixed time.

In this work, we establish a uniqueness result of the coefficient identification problem using Carleman estimates and particular complex geometrical solutions of the fractional diffusion equation. These techniques are inspired by [7], where the authors proved the uniqueness of a coefficient identification problem for the Schrödinger equation.

In Sect. 2, we state the forward problem. In Sect. 3, we recall some definitions and properties of the fractional derivatives, and in Sect. 4, we give the proof of our main result.

\section{Forward problem}

In this work, our fundamental question is to prove the uniqueness of the potential $q$ via the fractional diffusion equation from the knowledge of the Cauchy data. The forward problem is to find the solution $u$ from the following system of equations:

$$
\begin{cases}\partial_{t}^{\alpha} u-\Delta_{x} u+q(x) u=0 & \text { in } \Omega_{T}:=\Omega \times(0, T), \\ u(x, 0)=u_{0}(x) & \text { in } \Omega, \\ u=f & \text { on } \Sigma_{T}:=\partial \Omega \times(0, T)\end{cases}
$$

where the coefficient $q \in L^{\infty}(\bar{\Omega}), f \in C^{1}\left([0 ; T] ; H^{\frac{3}{2}}(\partial \Omega)\right)$, and $u_{0} \in L^{2}(\Omega)$ are given. Referring to $[17,20]$, we can choose $G \in C^{1}\left([0 ; T] ; H^{2}(\Omega)\right)$ satisfying $G=f$ on $\Sigma_{T}$, and by setting $w=u-G, w$ is a solution of

$$
\begin{cases}\partial_{t}^{\alpha} w-\Delta_{x} w+q(x) w=F & \text { in } \Omega_{T} \\ w(x, 0)=a(x) & \text { in } \Omega, \\ w=0 & \text { on } \Sigma_{T}\end{cases}
$$

where $F=-\left(\partial_{t}^{\alpha} G-\Delta_{x} G+q(x) G\right)$ and $a(x)=u_{0}(x)-G(x, 0)$. The study of the existence and uniqueness of the solution of (2.1) is reduced to the existence and uniqueness of problem (2.2). 
Theorem 2.1 Let $a \in L^{2}(\Omega)$ and $F \in L^{\infty}\left(0, T, L^{2}(\Omega)\right)$. Then problem (2.2) has a unique weak solution

$$
w \in C\left([0, T] ; L^{2}(\Omega)\right) \cap C\left((0, T] ; H^{2}(\Omega) \cap H_{0}^{1}(\Omega)\right) \cap L^{2}\left([0, T] ; H^{2}(\Omega) \cap H_{0}^{1}(\Omega)\right) .
$$

Proof We split problem (2.2) into the following two problems by taking $w=w_{1}+w_{2}$, where $w_{1}$ is the solution of

$$
\begin{cases}\partial_{t}^{\alpha} u-\Delta_{x} u+q(x) u=0 & \text { in } \Omega_{T}, \\ u(x, 0)=a & \text { in } \Omega, \\ u=0 & \text { on } \Sigma_{T},\end{cases}
$$

and $w_{2}$ is the solution of

$$
\begin{cases}\partial_{t}^{\alpha} u-\Delta_{x} u+q(x) u=F & \text { in } \Omega_{T}, \\ u(x, 0)=0 & \text { in } \Omega, \\ u=0 & \text { on } \Sigma_{T} .\end{cases}
$$

From Sakamoto et al. [22] we have

1. Problem (2.3) has a unique weak solution

$$
w_{1} \in C\left([0, T] ; L^{2}(\Omega)\right) \cap C\left((0, T] ; H^{2}(\Omega) \cap H_{0}^{1}(\Omega)\right)
$$

satisfying

$$
\|u(\cdot, t)\|_{H^{2}(\Omega)}+\left\|\partial_{t}^{\alpha} u(\cdot, t)\right\|_{L^{2}(\Omega)} \leq C_{1} t^{-\alpha}\|a\|_{L^{2}(\Omega)} .
$$

2. Problem (2.4) has a unique weak solution $w_{2} \in L^{2}\left([0, T] ; H^{2}(\Omega) \cap H_{0}^{1}(\Omega)\right)$ satisfying

$$
\|u\|_{L^{2}\left([0, T] ; H^{2}(\Omega)\right)}+\left\|\partial_{t}^{\alpha} u\right\|_{L^{2}\left(\Omega_{T}\right)} \leq C_{2}\|F\|_{L^{2}\left(\Omega_{T}\right)} .
$$

\section{Preliminaries}

We start this section by giving some definitions and fundamental properties of fractional integrals and fractional derivatives, which can be found in $[18,21]$.

Let $\alpha>0$, let $n$ be the integer satisfying $n-1 \leq \alpha<n$, and let $a, b \in \mathbb{R}$.

Definition 3.1 Let $g:[a, b] \rightarrow \mathbb{R}$ be a function, and let $\Gamma$ be the Euler gamma function.

1. The left and right Riemann-Liouville fractional integrals of order $\alpha$ are defined respectively by

$$
{ }_{a} I_{t}^{\alpha} g(t):=\frac{1}{\Gamma(\alpha)} \int_{a}^{t}(t-s)^{\alpha-1} g(s) d s
$$

and

$$
{ }_{t} I_{b}^{\alpha} g(t):=\frac{1}{\Gamma(\alpha)} \int_{t}^{b}(s-t)^{\alpha-1} g(s) d s .
$$


2. The left and right Riemann-Liouville fractional derivatives of order $\alpha$ are defined respectively by

$$
{ }_{a} D_{t}^{\alpha} g(t):=\frac{d^{n}}{d t^{n}}{ }_{a} I_{t}^{n-\alpha} g(t)=\frac{1}{\Gamma(n-\alpha)} \frac{d^{n}}{d t^{n}} \int_{a}^{t}(t-s)^{n-\alpha-1} g(s) d s
$$

and

$$
{ }_{t} D_{b}^{\alpha} g(t):=(-1)^{n} \frac{d^{n}}{d t^{n}}{ }_{t} I_{b}^{n-\alpha} g(t)=\frac{(-1)^{n}}{\Gamma(n-\alpha)} \frac{d^{n}}{d t^{n}} \int_{t}^{b}(s-t)^{n-\alpha-1} g(s) d s
$$

In particular, if $\alpha=0$, then

$$
{ }_{a} D_{t}^{0} g(t)={ }_{t} D_{b}^{0} g(t)=g(t)
$$

and if $\alpha=k \in \mathbb{N}$, then

$$
{ }_{a} D_{t}^{k} g(t)={ }_{t} D_{b}^{k} g(t)=g^{(k)}(t)
$$

3. The left and right Caputo fractional derivatives of order $\alpha$ are defined by

$$
{ }_{a}^{c} D_{t}^{\alpha} g(t):={ }_{a} I_{t}^{n-\alpha} g^{(n)}(t)=\frac{1}{\Gamma(n-\alpha)} \int_{a}^{t}(t-s)^{n-\alpha-1} g^{(n)}(s) d s
$$

and

$$
{ }_{t}^{c} D_{b}^{\alpha} g(t):=(-1)_{t}^{n} I_{b}^{n-\alpha} g^{(n)}(t)=\frac{(-1)^{n}}{\Gamma(n-\alpha)} \int_{t}^{b}(s-t)^{n-\alpha-1} g^{(n)}(s) d s .
$$

In particular, if $0<\alpha<1$, then we denote

$$
\partial_{t}^{\alpha} g(t):={ }_{0}^{c} D_{t}^{\alpha} g(t)=\frac{1}{\Gamma(1-\alpha)} \int_{0}^{t}(t-s)^{-\alpha} g^{\prime}(s) d s .
$$

Lemma 3.2 ([18]) If $g \in A C^{n}[a, b]$, then the Riemann-Liouville fraction derivative and the Caputo fractional derivative are connected with each other by the following relations:

$$
{ }_{a} D_{t}^{\alpha} g(t)={ }_{0}^{c} D_{t}^{\alpha} g(t)+\sum_{k=0}^{n-1} \frac{g^{(k)}(a)}{\Gamma(1+k-\alpha)}(t-a)^{k-\alpha}
$$

and

$$
{ }_{t} D_{b}^{\alpha} g(t)={ }_{0}^{c} D_{b}^{\alpha} g(t)+\sum_{k=0}^{n-1} \frac{(-1)^{k} g^{(k)}(b)}{\Gamma(1+k-\alpha)}(b-t)^{k-\alpha} .
$$

Here

$$
A C^{n}[a, b]=\left\{g:[a, b] \rightarrow \mathbb{R} \text { such that } \frac{d^{n-1}}{d x^{n-1}}(g) \in A C[a, b]\right\},
$$




$$
g \in A C[a, b] \quad \Leftrightarrow \quad \text { there exists } \varphi \in L(a, b) \text { such that } g(x)=c+\int_{a}^{b} \varphi(t) d t, \quad c \in \mathbb{R}
$$

and $L(a, b)$ is the set of Lebesgue-measurable complex-valued functions on $[a, b]$.

Remark 3.3 If $0<\alpha<1$, then

$$
{ }_{0} D_{t}^{\alpha} u(x, t)={ }_{0}^{c} D_{t}^{\alpha} u(x, t)+\frac{u(x, 0)}{\Gamma(1-\alpha)} t^{-\alpha}
$$

In addition, if $u(x, 0)=0$, then ${ }_{0} D_{t}^{\alpha} u(x, t)={ }_{0}^{c} D_{t}^{\alpha} u(x, t)$.

Definition 3.4 ([18])

1. The Mittag-Leffler function is

$$
E_{\alpha, \beta}(z)=\sum_{k=0}^{\infty} \frac{z^{k}}{\Gamma(\alpha k+\beta)}, \quad z \in \mathbb{C}
$$

where $\alpha>0$ and $\beta$ are arbitrary constants.

2. The $\alpha$-exponential function is defined by

$$
e_{\alpha}^{\lambda z}=z^{\alpha-1} E_{\alpha, \alpha}(\lambda z), \quad z \in \mathbb{C} \backslash\{0\},
$$

where $\alpha>0$ and $\lambda \in \mathbb{C}$.

\section{Lemma 3.5 ([18])}

1. For $0<\alpha<1$ and $\lambda>0$, we have

$$
\partial_{t}^{\alpha} E_{\alpha, 1}\left(-\lambda t^{\alpha}\right)=-\lambda E_{\alpha, 1}\left(-\lambda t^{\alpha}\right), \quad t>0
$$

2. For $0<\alpha<1$ and $\lambda \in \mathbb{C}$, we have

$$
{ }_{0} D_{t}^{\alpha} e_{\alpha}^{\lambda t}=\lambda e_{\alpha}^{\lambda t}, \quad t>0 .
$$

Lemma 3.6 ([21]) For $s>|\lambda|^{\frac{1}{\alpha}}$, we have

$$
\int_{0}^{\infty} e^{-s t} t^{\alpha k+\beta-1} E_{\alpha, \beta}^{(k)}\left(-\lambda t^{\alpha}\right) d t=\frac{k ! s^{\alpha-\beta}}{\left(s^{\alpha}-\lambda\right)^{k+1}}
$$

\section{The main result}

In this work, we focus on the uniqueness of the solution $q$ of the inverse problem.

Let

$$
H_{\Delta}\left(\Omega_{T}\right)=\left\{u \in \mathcal{D}^{\prime}\left(\Omega_{T}\right) / u \in L^{2}\left(\Omega_{T}\right), \Delta u \in L^{2}\left(\Omega_{T}\right)\right\} .
$$

For $x=\left(x_{1}, \ldots, x_{d}\right)$ and $y=\left(y_{1}, \ldots, y_{d}\right) \in \mathbb{C}^{d},\langle x, y\rangle:=\sum_{i=1}^{d} x_{i} y_{i}$.

Fix $\xi \in S^{d-1}$, where $S^{d-1}=\left\{x \in \mathbb{R}^{d},|x|=1\right\}$, and for $\varepsilon>0$, denote

$$
\partial \Omega_{+}(\xi)=\{x \in \partial \Omega,\langle v(x), \xi\rangle>0\}
$$




$$
\begin{aligned}
& \partial \Omega_{-}(\xi)=\{x \in \partial \Omega,\langle v(x), \xi\rangle<0\}, \\
& \partial \Omega_{+, \varepsilon}(\xi)=\{x \in \partial \Omega,\langle v(x), \xi\rangle>\varepsilon\}, \\
& \partial \Omega_{-, \varepsilon}(\xi)=\{x \in \partial \Omega,\langle v(x), \xi\rangle<\varepsilon\},
\end{aligned}
$$

where $v$ represents the unit outer normal to the boundary $\partial \Omega$,

$$
C_{q, \varepsilon}=\left\{\left(u(\cdot, 0), u_{\mid \Sigma_{T}}, \partial_{\nu} u_{\mid \partial \Omega_{-, \varepsilon}(\xi) \times(0, T)}\right) / u \in H_{\Delta}\left(\Omega_{T}\right),\left(\partial_{t}^{\alpha}-\Delta+q\right) u=0 \text { in } \Omega_{T}\right\} .
$$

In the following theorem, we give a global uniqueness result for the fractional diffusion equation in which the Cauchy data are given only on part of the boundary.

Theorem 4.1 Let $q_{i} \in L^{\infty}(\Omega), i=1$, 2. Given $\xi \in S^{d-1}$ and $\varepsilon>0$, assume that $C_{q_{1}, \varepsilon}=C_{q_{2}, \varepsilon}$. Then $q_{1}=q_{2}$.

To prove this result, we need the following results.

Lemma 4.2 ([7]) Let $\rho \in \mathbb{C}^{d}$ be such that $\langle\rho, \rho\rangle=0$, where $\rho=\tau(\xi+i \eta)$ with $\xi, \eta \in S^{d-1}$. Suppose that $f(\cdot, \rho /|\rho|) \in W^{2, \infty}(\Omega)$ satisfies $\partial_{\xi} f=\partial_{\eta} f=0$, where $\partial_{\xi}$ denotes the directional derivative in the direction $\xi$. Then there is a solution to $\Delta u-q u=0$ in $\Omega$ of the form

$$
u(x, \rho)=e^{\langle x, \rho\rangle}(f(x, \rho /|\rho|)+\psi(x, \rho))
$$

where

$$
\psi_{\mid \partial \Omega_{-}(\xi)}=0
$$

and

$$
\|\psi(\cdot, \rho)\|_{L^{2}(\Omega)} \leq \frac{M}{\tau}
$$

for some $M>0$ and $\tau \geq \tau_{0}>0$.

Corollary 4.3 Let $\rho \in \mathbb{C}^{d}$ be such that $\langle\rho, \rho\rangle=0$, where $\rho=\tau(\xi+i \eta)$ with $\xi, \eta \in S^{d-1}$, and $\lambda>0$.

1. There is a solution $w$ to $-\Delta w+(q-\lambda) w=0$ in $\Omega$ of the form

$$
u_{q, \lambda}(x, \rho):=e^{\langle x, \rho\rangle}\left(1+\psi_{q}(x, \rho)\right)
$$

where

$$
\psi_{q \mid \partial \Omega_{-}(\xi)}=0
$$

and

$$
\left\|\psi_{q}(\cdot, \rho)\right\|_{L^{2}(\Omega)} \leq \frac{M}{\tau}, \quad \tau \geq \tau_{0},
$$

for some $M>0$ and $\tau_{0}>0$. 
2. The function $(x, t) \mapsto E_{\alpha, 1}\left(-\lambda t^{\alpha}\right) u_{q, \lambda}(x, \rho)$ is a solution of $\left(\partial_{t}^{\alpha}-\Delta+q\right) w=0$ in $\Omega_{T}$.

3. The function $(x, t) \mapsto e_{\alpha}^{-\lambda t} u_{q, \lambda}(x, \rho)$ is a solution of $\left({ }_{0} D_{t}^{\alpha}-\Delta+q\right) w=0$ in $\Omega_{T}$.

Proof 1. Applying Lemma 4.2 for $f=1$ and $q:=q-\lambda$, we obtain the result.

2. Using Proposition 3.5 and item 1 , it is easy to prove items 2 and 3.

Lemma 4.4 (Carleman estimates [7]) For $q \in L^{\infty}(\Omega)$, there exist $\tau_{0}>0$ and $C>0$ such that for all $u \in \mathcal{C}^{2}(\Omega), u_{\mid \partial \Omega}=0$, and $\tau \geq \tau_{0}$, we have the estimate

$$
\begin{aligned}
& C \tau^{2} \int_{\Omega}\left|e^{-\tau\langle x, \xi\rangle} u\right|^{2} d x+\tau \int_{\partial \Omega_{+}}\langle\nu, \xi\rangle\left|e^{-\tau\langle x, \xi\rangle} \partial_{\nu} u\right|^{2} d S \\
& \quad \leq \int_{\Omega}\left|e^{-\tau\langle x, \xi\rangle}(\Delta-q) u\right|^{2} d x-\tau \int_{\partial \Omega_{-}}\langle\nu, \xi\rangle\left|e^{-\tau\langle x, \xi\rangle} \partial_{\nu} u\right|^{2} d S .
\end{aligned}
$$

Lemma 4.5 If $u \in H_{\Delta}\left(\Omega_{T}\right)$ and $u_{\mid \Sigma_{T}}=0$, then $u \in L^{2}\left(0, T ; H^{2}(\Omega)\right)$.

Proof Suppose first that $u \in \mathcal{C}^{2}\left(\bar{\Omega}_{T}\right)$. From the first Green formula we obtain

$$
2 \int_{\Omega_{T}}|\nabla u|^{2} d x=-2 \int_{\Omega_{T}} u \Delta u d x \leq \int_{\Omega_{T}}\left(|u|^{2}+|\Delta u|^{2}\right) d x=\|u\|_{H_{\Delta}\left(\Omega_{T}\right)}^{2}<\infty .
$$

From the formula of Kadlec [24],

$$
\sum_{i, j} \int_{\Omega_{T}}\left|\partial_{i} \partial_{j} u\right|^{2} d x \leq \int_{\Omega_{T}}|\Delta u|^{2} d x+C_{0} \int_{\Sigma_{T}}\left|\partial_{\nu} u\right|^{2} d x
$$

since $\int_{\Sigma_{T}}\left|\partial_{\nu} u\right|^{2} d x \leq C_{1} \int_{\Omega_{T}}|\Delta u|^{2} d x$, we get

$$
\|u\|_{L^{2}\left(0, T ; H^{2}(\Omega)\right)} \leq C_{2}\|u\|_{H_{\Delta}\left(\Omega_{T}\right)} .
$$

Using standard density arguments, we obtain

$$
\|u\|_{L^{2}\left(0, T ; H^{2}(\Omega)\right)} \leq C_{2}\|u\|_{H_{\Delta}\left(\Omega_{T}\right)}, \quad \forall u \in H_{\Delta}\left(\Omega_{T}\right) \text {, and } u_{\mid \Sigma_{T}}=0 .
$$

Thus $u \in L^{2}\left(0, T ; H^{2}(\Omega)\right)$.

Lemma 4.6 Let $u_{i}:=u_{q_{i} f_{i}}^{\alpha}, i=1,2$, be solutions of the fractional diffusion equations $\left(P_{q_{i}, f_{i}}^{\alpha}\right)$, $q_{i} \in L^{\infty}(\Omega)$ and $f_{i} \in L^{2}\left(\Sigma_{T}\right)$. Then we have

$$
\partial_{t}^{\alpha} u_{1} \star u_{2}=u_{1} \star_{0} D_{t}^{\alpha} u_{2},
$$

where $\star$ is the convolution product.

Proof From [2] we have

$$
\int_{a}^{b} g(s)_{a}^{c} D_{s}^{\alpha} f(s) d s=\int_{a}^{b} f(s)_{s} D_{b}^{\alpha} g(s) d s+\sum_{j=0}^{n-1}\left[{ }_{s} D_{b}^{\alpha+j-n} g(s) \cdot{ }_{s} D_{b}^{n-1-j} f(s)\right]_{a}^{b}
$$


for $0<\alpha<1, a=0$, and $b=t$, and

$$
\int_{0}^{t} g(s) \partial_{s}^{\alpha} f(s) d s=\int_{0}^{t} f(s)_{s} D_{t}^{\alpha} g(s) d s+\left[{ }_{s} D_{t}^{\alpha-1} g(s) \cdot f(s)\right]_{0}^{t} .
$$

Then

$$
\begin{aligned}
\partial_{t}^{\alpha} u_{1} \star u_{2} & =\int_{0}^{t} \partial_{s}^{\alpha} u_{1}(x, s) u_{2}(x, t-s) d s \\
& =\int_{0}^{t} u_{1}(x, s)_{s} D_{t}^{\alpha} u_{2}(x, t-s) d s+\left[{ }_{s} D_{t}^{\alpha-1} u_{2}(x, t-s) u_{1}(x, s)\right]_{0}^{t}
\end{aligned}
$$

Since $\alpha-1<0$, we have ${ }_{s} D_{t}^{\alpha-1} u_{2}(x, t-s)={ }_{s} I_{t}^{1-\alpha} u_{2}(x, t-s)$ (see [2]) and

$$
{ }_{s} I_{t}^{1-\alpha} u_{2}(x, t-s)=\frac{1}{\Gamma(1-\alpha)} \int_{s}^{t}(\tau-s)^{-\alpha} u_{2}(x, t-\tau) d \tau \text {. }
$$

Using integration by parts, we get

$$
{ }_{s} I_{t}^{1-\alpha} u_{2}(x, t-s)=\frac{1}{\Gamma(2-\alpha)}\left[(t-s)^{1-\alpha} u_{2}(x, t-s)+\int_{s}^{t}(\tau-s)^{1-\alpha} \frac{\partial u_{2}}{\partial \tau}(x, t-\tau) d \tau\right]
$$

We deduce that $\lim _{s \rightarrow t s} D_{t}^{\alpha-1} u_{2}(x, t-s)=0$ and

$$
\int_{0}^{t} \partial_{s}^{\alpha} u_{1}(x, s) u_{2}(x, t-s) d s=\int_{0}^{t} u_{1}(x, s)_{s} D_{t}^{\alpha} u_{2}(x, t-s) d s
$$

By definition

$$
{ }_{s} D_{t}^{\alpha} u_{2}(x, t-s):=\frac{1}{\Gamma(1-\alpha)} \frac{d}{d t} \int_{s}^{t}(t-\tau)^{-\alpha} u_{2}(x, \tau-s) d \tau \text {. }
$$

By change of variable $\xi=\tau-s$ we obtain

$$
\begin{aligned}
{ }_{s} D_{t}^{\alpha} u_{2}(x, t-s) & =\frac{1}{\Gamma(1-\alpha)} \frac{d}{d t} \int_{0}^{t-s}(t-s-\xi)^{-\alpha} u_{2}(x, \xi) d \xi \\
& ={ }_{0} D_{t-s}^{\alpha} u_{2}(x, t-s),
\end{aligned}
$$

which implies

$$
\int_{0}^{t} \partial_{s}^{\alpha} u_{1}(x, s) u_{2}(x, t-s) d s=\int_{0}^{t} u_{1}(x, s)_{0} D_{t-s}^{\alpha} u_{2}(x, t-s) d s
$$

and

$$
\partial_{t}^{\alpha} u_{1} \star u_{2}=u_{1} \star_{0} D_{t}^{\alpha} u_{2}
$$

Remark 4.7 If $u_{2}(x, 0)=0$, then from Remark 3.3 we obtain

$$
\partial_{t}^{\alpha} u_{1} \star u_{2}=u_{1} \star \partial_{t}^{\alpha} u_{2}
$$


Proof of Theorem 4.1 Let $\xi \in S^{d-1}$. Fix $k \in \mathbb{R}^{d}$ such that $\langle k, \xi\rangle=0$. By Corollary 4.3 there exists $u_{2} \in H_{\Delta}\left(\Omega_{T}\right)$ satisfying $\left(\partial_{t}^{\alpha}-\Delta+q_{2}\right) u_{2}=0$ in $\Omega_{T}$ of the form

$$
u_{2}(x, t)=E_{\alpha}\left(-\lambda t^{\alpha}\right) u_{q_{2}, \lambda}\left(x, \rho_{2}\right)
$$

where $u_{q_{2}, \lambda}$ id defined by (4.2), $\rho_{2}=\tau \xi-i \frac{k+\ell}{2},\langle k, \ell\rangle=\langle\xi, \ell\rangle=0$, and $|k+\ell|^{2}=4 \tau^{2}$ (under these conditions, $\left\langle\rho_{2}, \rho_{2}\right\rangle=0$ ). In dimension $d \geq 3$, we can always choose such a vector $\ell$.

Since $C_{q_{1}, \varepsilon}=C_{q_{2}, \varepsilon}$, there exists $u_{1} \in H_{\Delta}\left(\Omega_{T}\right)$ such that

$$
\begin{cases}\left(\partial_{t}^{\alpha}-\Delta+q_{1}\right) u_{1}=0 & \text { in } \Omega_{T}, \\ u_{1}(x, 0)=u_{2}(x, 0) & \text { in } \Omega, \\ u_{1}=u_{2} & \text { on } \Sigma_{T}, \\ \partial_{\nu} u_{1}=\partial_{\nu} u_{2} & \text { on } \partial \Omega_{-, \varepsilon}(\xi) \times(0, T) .\end{cases}
$$

Setting $u=u_{1}-u_{2}$ and $q=q_{1}-q_{2}$, we have

$$
\begin{cases}\left(\partial_{t}^{\alpha}-\Delta+q_{1}\right) u=-q u_{2} & \text { in } \Omega_{T}, \\ u(x, 0)=0 & \text { in } \Omega, \\ u=0 & \text { on } \Sigma_{T}, \\ \partial_{\nu} u=0 & \text { on } \partial \Omega_{-, \varepsilon}(\xi) \times(0, T) .\end{cases}
$$

By Lemma 4.5, since $u \in H_{\Delta}\left(\Omega_{T}\right)$ and $u_{\mid \Sigma_{T}}=0$, it follows that $u \in L^{2}\left(0, T ; H^{2}(\Omega)\right)$.

From Green's formula and Lemma 4.6, for $v \in H_{\Delta}\left(\Omega_{T}\right)$, we obtain

$$
\begin{aligned}
\int_{\Omega}\left(\partial_{t}^{\alpha}-\Delta+q_{1}\right) u \star \bar{v} d x & =-\int_{\Omega} q u_{2} \star \bar{v} d x \\
& =\int_{\Omega} u \star\left({ }_{0} D_{t}^{\alpha}-\Delta+q_{1}\right) \bar{v} d x+\int_{\partial \Omega} \partial_{\nu} u \star \bar{v} d s
\end{aligned}
$$

We choose

$$
\bar{v}(x, t)=e_{\alpha}^{-\lambda t} u_{q_{1}, \lambda}\left(x, \rho_{1}\right),
$$

which is a solution of $\left({ }_{0} D_{t}^{\alpha}-\Delta+q_{1}\right) \bar{v}=0$, where $u_{q_{1}, \lambda}\left(x,, \rho_{1}\right)=e^{\left\langle x, \rho_{1}\right\rangle}\left(1+\psi_{q_{1}}\left(x, \rho_{1}\right)\right)$,

$$
\psi_{q_{1} \mid \partial \Omega_{-}(\xi)}=0
$$

and

$$
\left\|\psi_{q_{1}}\left(\cdot, \rho_{1}\right)\right\|_{L^{2}(\Omega)} \leq \frac{\tilde{M}}{\tau}, \quad \tau \geq \tau_{0},
$$

where $\rho_{1}=-\tau \xi-i \frac{k-\ell}{2}$. We have $\rho_{1}+\rho_{2}=-i k$ and $\left|\rho_{1}\right|^{2}=2 \tau^{2}$.

Then equation (4.9) becomes

$$
-\int_{\Omega} q u_{2} \star \bar{v} d x=\int_{\partial \Omega} \partial_{\nu} u \star \bar{v} d s .
$$


Let $\lambda>0$ and $s>\lambda^{\frac{1}{\alpha}}$, extending $u$ by 0 on $(T, \infty)$. Applying the Laplace transform to (4.10), we obtain

$$
-\int_{\Omega} q \tilde{u_{2}} \tilde{\bar{v}} d x=\int_{\partial \Omega} \partial_{\nu} \tilde{\bar{u}} \tilde{\bar{v}} d S
$$

where $\tilde{u}:=\tilde{u}(\cdot, s)$ is a solution of the following problem:

$$
\begin{cases}\Delta \tilde{u}-\left(q_{1}+s^{\alpha}\right) \tilde{u}=q \tilde{u}_{2} & \text { in } \Omega, \\ \tilde{u}=0 & \text { on } \partial \Omega, \\ \partial_{\nu} \tilde{u}=0 & \text { on } \partial \Omega_{-, \varepsilon}(\xi),\end{cases}
$$

and the Laplace transform $\tilde{g}$ of a function $g$ is defined by

$$
\tilde{g}(s)=\int_{0}^{\infty} e^{-s t} g(t) d t
$$

Using Lemma 3.6, we obtain

$$
\begin{aligned}
& \tilde{u_{2}}(x, s)=\frac{s^{\alpha-1}}{s^{\alpha}-\lambda} u_{q_{2}, \lambda}\left(x, \rho_{2}\right), \\
& \tilde{\bar{v}}(x, s)=\frac{1}{s^{\alpha}-\lambda} u_{q_{1}, \lambda}\left(x, \rho_{1}\right) .
\end{aligned}
$$

Equation (4.11) becomes

$$
\int_{\Omega} q u_{q_{1}, \lambda}\left(x, \rho_{1}\right) u_{q_{2}, \lambda}\left(x, \rho_{2}\right) d x=\frac{\left(s^{\alpha}-\lambda\right)}{s^{\alpha-1}} \int_{\partial \Omega} \partial_{\nu} \tilde{u} u_{q_{1}, \lambda}\left(x, \rho_{1}\right) d S .
$$

In the following step, we show that $\lim _{\tau \rightarrow \infty} \int_{\partial \Omega} \partial_{\nu} \tilde{u} u_{q_{1}, \lambda}\left(x, \rho_{1}\right) d S=0$. Indeed, since $\partial_{\nu} \tilde{u}=0$ on $\partial \Omega_{-, \varepsilon}(\xi)$, we have

$$
\begin{aligned}
& \left|\int_{\partial \Omega} \partial_{\nu} \tilde{u} u_{q_{1}, \lambda}\left(x, \rho_{1}\right) d S\right| \\
& \quad=\left|\int_{\partial \Omega_{+, \varepsilon}(\xi)} \partial_{\nu} \tilde{u} e^{\left\langle x, \rho_{1}\right\rangle}\left(1+\psi_{q_{1}}\left(x, \rho_{1}\right)\right) d S\right| \\
& \quad \leq \int_{\partial \Omega_{+, \varepsilon}(\xi)}\left|\partial_{\nu} \tilde{u} e^{-\tau\langle x, \xi\rangle}\right|\left|1+\psi_{q_{1}}\left(x, \rho_{1}\right)\right| d S \\
& \quad \leq\left(\int_{\partial \Omega_{+, \varepsilon}(\xi)}\left|\partial_{\nu} \tilde{u} e^{-\tau\langle x, \xi\rangle}\right|^{2} d S\right)^{\frac{1}{2}}\left(\int_{\partial \Omega_{+, \varepsilon}(\xi)}\left|1+\psi_{q_{1}}\left(x, \rho_{1}\right)\right|^{2} d S\right)^{\frac{1}{2}} \\
& \quad \leq\left(\int_{\partial \Omega_{+, \varepsilon}(\xi)}\left|\partial_{\nu} \tilde{u} e^{-\tau\langle x, \xi\rangle}\right|^{2} d S\right)^{\frac{1}{2}}\left(2 \int_{\partial \Omega_{+, \varepsilon}(\xi)}\left(1+\left|\psi_{q_{1}}\left(x, \rho_{1}\right)\right|^{2}\right) d S\right)^{\frac{1}{2}} \\
& \quad \leq \sqrt{2}\left(\int_{\partial \Omega_{+, \varepsilon}(\xi)}\left|\partial_{\nu} \tilde{u} e^{-\tau\langle x, \xi\rangle}\right|^{2} d S\right)^{\frac{1}{2}}\left(\operatorname{vol}(\partial \Omega)+\left\|\psi_{q_{1}}\right\|_{L^{2}(\partial \Omega)}^{2}\right)^{\frac{1}{2}} .
\end{aligned}
$$

From [7, p. 666] we have

$$
\left\|\psi_{q_{1}}\right\|_{\mathcal{C}(\partial \Omega)} \leq C \tau^{\frac{1}{4}}
$$


and since

$$
\left\|\psi_{q_{1}}\right\|_{L^{2}(\partial \Omega)} \leq \operatorname{vol}(\partial \Omega)\left\|\psi_{q_{1}}\right\|_{\mathcal{C}(\partial \Omega)},
$$

we have

$$
\left|\int_{\partial \Omega} \partial_{\nu} \tilde{u} u_{q_{1}, \lambda}\left(x, \rho_{1}\right) d S\right| \leq C \sqrt{2}(\operatorname{vol}(\partial \Omega))^{\frac{1}{2}}\left(1+\tau^{\frac{1}{4}}\right)\left(\int_{\partial \Omega_{+, \varepsilon}(\xi)}\left|\partial_{\nu} \tilde{u} e^{-\tau\langle x, \xi\rangle}\right|^{2} d S\right)^{\frac{1}{2}} .
$$

From estimates (4.3) and (4.1) we obtain

$$
\begin{aligned}
\tau \varepsilon \int_{\partial \Omega_{+, \varepsilon}(\xi)}\left|\partial_{\nu} \tilde{u} e^{-\tau\langle x, \xi\rangle}\right|^{2} d s & \leq \tau \int_{\partial \Omega_{+, \varepsilon}(\xi)}\langle v, \xi\rangle\left|\partial_{\nu} \tilde{u} e^{-\tau\langle x, \xi\rangle}\right|^{2} d S \\
& \leq \int_{\Omega}\left|e^{-\tau\langle x, \xi\rangle}\left(\Delta-\left(q_{1}+s^{\alpha}\right)\right) \tilde{u}\right|^{2} d x=\int_{\Omega}\left|e^{-\tau\langle x, \xi\rangle} q \tilde{u}_{2}\right|^{2} d x \\
& \leq 2\left(\left\|q_{1}\right\|_{\infty}+\left\|q_{2}\right\|_{\infty}\right)^{2} \int_{\Omega}\left(1+\left|\psi_{q_{2}}\left(x, \rho_{2}\right)\right|^{2}\right) d x \\
& \leq 2\left(\left\|q_{1}\right\|_{\infty}+\left\|q_{2}\right\|_{\infty}\right)^{2}\left(\operatorname{vol}(\Omega)+\left\|\psi_{q_{2}}\left(x, \rho_{2}\right)\right\|_{L^{2}(\Omega)}^{2}\right) \\
& \leq 2\left(\left\|q_{1}\right\|_{\infty}+\left\|q_{2}\right\|_{\infty}\right)^{2}\left(\operatorname{vol}(\Omega)+\frac{M}{\tau^{2}}\right) .
\end{aligned}
$$

Hence we have proved that

$$
\left|\int_{\partial \Omega} \partial_{\nu} \tilde{u} \bar{v} d s\right| \leq \tilde{C} \frac{\left(1+\tau^{\frac{1}{4}}\right)}{\sqrt{\tau}}\left(\sqrt{\operatorname{vol}(\Omega)}+\frac{M}{\tau}\right),
$$

where

$$
\tilde{C}=\frac{2 C\left(\left\|q_{1}\right\|_{\infty}+\left\|q_{2}\right\|_{\infty}\right) \sqrt{\operatorname{vol}(\partial \Omega)}}{\sqrt{\varepsilon}},
$$

and then

$$
\lim _{\tau \rightarrow \infty} \int_{\partial \Omega} \partial_{\nu} \tilde{u} \tilde{\bar{v}} d S=0 .
$$

Using equalities (4.1) and (4.11), by tending $\tau$ to $\infty$ we have

$$
\int_{\Omega} q(x) e^{-i\langle x, k\rangle} d x=0
$$

Changing $\xi \in S^{d-1}$ in a small conic neighborhood and using the fact that $\hat{q}(k)$ is analytic, we get that $q=0$, and then $q_{1}=q_{2}$.

\section{Conclusion}

In this paper, we established a uniqueness result for the coefficient identification problem in a fractional diffusion equation in a bounded domain from the observation of the Cauchy data on particular subsets of the boundary using Carleman estimates and particular complex geometrical solutions of the fractional diffusion equation. 
Acknowledgements

Not applicable.

\section{Funding}

Not applicable.

\section{Abbreviations}

Not applicable.

Availability of data and materials

Not applicable.

\section{Competing interests}

The authors declare that there is no conflict of interest regarding the publication of this paper.

\section{Authors' contributions}

All authors contributed equally to this paper. All authors read and approved the final manuscript.

\section{Author details}

'Inverse Problems, LAMSIN, Ecole Nationale d'Ingénieurs de Tunis, University of Tunis El Manar, Tunis Belvédère, Tunisia.

${ }^{2}$ Mathematics Department, Jamoum University College, Umm Al-Qura University, Mecca, Saudi Arabia. ${ }^{3}$ Mathematics

Department, Faculty of Science and Arts, Khulais, University of Jeddah, Jeddah, Saudi Arabia.

\section{Publisher's Note}

Springer Nature remains neutral with regard to jurisdictional claims in published maps and institutional affiliations.

Received: 21 February 2019 Accepted: 9 October 2019 Published online: 26 October 2019

References

1. Adams, E.E., Gelhar, L.W.: Field study of dispersion in a heterogeneous aquifer 2. Spatial moments analysis. Water Resour. Res. 28, 3293-3307 (1992)

2. Agrawal, O.P.: Fractional variational calculus in terms of Riesz fractional derivatives. J. Phys. A, Math. Theor. 40, 6287-6303 (2007)

3. Bellassoued, M., Choulli, M., Yamamoto, M.: Stability estimate for an inverse wave equation and a multidimensional Borg-Levinson theorem. J. Differ. Equ. 247(2), 465-494 (2009)

4. Bellassoued, M., Dos Santos Ferreira, D.: Stability estimates for the anisotropic wave equation from the Dirichlet-to-Neumann map. Inverse Probl. Imaging 5(4), 745-773 (2011)

5. Bellassoued, M., Jellali, D., Yamamoto, M.: Lipschitz stability for a hyperbolic inverse problem by finite local boundary data. Appl. Anal. 85, 1219-1243 (2006)

6. Ben Aicha, I.: Stability estimate for hyperbolic inverse problem with time dependent coefficient. Inverse Probl. 31, $125010(2015)$

7. Bukhgeim, A.L., Uhlmann, G.: Recovering a potential from partial Cauchy data. Commun. Partial Differ. Equ. 27(3-4), 653-668 (2002)

8. Canuto, B., Kavian, O.: Determining coefficients in a class of heat equations via boundary measurements. SIAM J. Math. Anal. 32(5), 963-986 (2001)

9. Carcione, J., Sanchez-Sesma, F., Luzon, F., Perez Gavilan, J.: Theory and simulation of time-fractional fluid diffusion in porous media. J. Phys. A, Math. Theor. 46, 345501 (2013)

10. Cheng, J., Xiang, X., Yamamoto, M.: Carleman estimate for a fractional diffusion equation with half order and application. Appl. Anal. 90(9), 1355-1371 (2011)

11. Cheng, M., Nakagawa, J., Yamamoto, M., Yamazaki, T.: Uniqueness in an inverse problem for a one-dimensional fractional diffusion equation. Inverse Probl. 25, 115002 (2009)

12. Choulli, M.: Une Introduction aux Problèmes Inverses Elliptiques et Paraboliques. Springer, Berlin (2009)

13. Choulli, M., Kian, Y.: Stability of the determination of a time-dependent coefficient in parabolic equations. Math. Control Relat. Fields 3(2), 143-160 (2013)

14. Fujishiro, K., Kian, Y.: Determination of time dependent factors of coefficients in fractional diffusion equations. Preprint. arXiv:1501.01945

15. Ghanmi, A., Mdimagh, R., Saad, I.B.: Identification of points sources via time fractional diffusion equation. Filomat 32 6189-6201 (2018)

16. Hatano, Y., Nakagawa, J., Wang, S., Yamamoto, M.: Determination of order in fractional diffusion equation. J. Math-for-Ind. 5A, 51-57 (2013)

17. Kian, Y., Oksanen, L., Soccorsi, E., Yamamoto, M.: Global uniqueness in an inverse problem for time fractional diffusion equations. J. Differ. Equ. 264(2), 1146-1170 (2018)

18. Kilbas, A.A., Srivastava, H.M., Trujillo, J.J.: Theory and Applications of Fractional Differential Equations. Elsevier, Amsterdam (2006)

19. Li, Z., Imanuvilov, O.Y., Yamamoto, M.: Uniqueness in inverse boundary value problems for fractional diffusion equations. Preprint. arXiv:1404.7024

20. Lions, J.L., Magenes, E.: Problèmes aux limites non homogènes et applications, vol. 1. Dunod, Paris (1968)

21. Podlubny, I.: Fractional Differential Equations. Mathematics in Science and Engineering, vol. 198. Academic Press, San Diego (1999)

22. Sakamoto, S., Yamamoto, M.: Initial value/boundary value problems for fractional diffusion-wave equations and applications to some inverse problems. J. Math. Anal. Appl. 382, 426-447 (2011) 
23. Stefanov, P., Uhlmann, G.: Recovery of a source term or a speed with one measurement and applications. Trans. Am. Math. Soc. 365(11), 5737-5758 (2013)

24. Taylor, M.: Partial Differential Equations I. Basic Theory, 2nd edn. Springer, New York (1996)

25. Yamamoto, M., Zhang, Y.: Conditional stability in determining a zeroth-order coefficient in a half-order fractional diffusion equation by a Carleman estimate. Inverse Probl. 28(10), 105010 (2012)

Submit your manuscript to a SpringerOpen ${ }^{\circ}$ journal and benefit from:

- Convenient online submission

$\checkmark$ Rigorous peer review

- Open access: articles freely available online

- High visibility within the field

- Retaining the copyright to your article

Submit your next manuscript at $\gg$ springeropen.com 